\title{
BMJ Open Protocol for a randomised controlled trial to compare postoperative complications between minimally invasive and open DIStal PAnCreaTectomy (DISPACT-2 trial)
}

\author{
Pascal Probst (D) ,1,2 Fabian Schuh,, ${ }^{1,2}$ Colette Dörr-Harim, ${ }^{2}$ Anja Sander, ${ }^{3}$ \\ Thomas Bruckner, ${ }^{3}$ Christina Klose, ${ }^{3}$ Inga Rossion, ${ }^{2}$ Felix Nickel, ${ }^{1}$ \\ Beat Peter Müller-Stich, ${ }^{1}$ Arianeb Mehrabi (D) , ${ }^{1}$ Thilo Hackert, ${ }^{1}$ Markus W. Büchler, \\ Markus K. Diener
}

To cite: Probst $P$, Schuh F Dörr-Harim C, et al. Protocol for a randomised controlled trial to compare postoperative complications between minimally invasive and open DIStal PAnCreaTectomy (DISPACT-2 trial). BMJ Open 2021;11:e047867. doi:10.1136/ bmjopen-2020-047867

- Prepublication history for this paper is available online. To view these files, please visit the journal online (http://dx.doi. org/10.1136/bmjopen-2020047867).

Received 10 December 2020 Revised 21 December 2020 Accepted 27 January 2021

\section{Check for updates}

(c) Author(s) (or their employer(s)) 2021. Re-use permitted under CC BY-NC. No commercial re-use. See rights and permissions. Published by BMJ.

For numbered affiliations see end of article.

Correspondence to Prof. Pascal Probst; pascal.probst@med.uniheidelberg.de

\section{ABSTRACT}

Introduction In recent years, minimally invasive distal pancreatectomy (MIDP) has been used with increasing frequency to accelerate patient recovery. Distal pancreatectomy has an overall morbidity rate of $30 \%-40 \%$. The known advantages of minimally invasive techniques must be rigorously compared with those of open surgery before they can be completely implemented into clinical practice.

Methods and analysis DISPACT- 2 is a multicentre randomised controlled trial comparing minimally invasive (conventional laparoscopic or robotic assisted) with open distal pancreatic resection in patients undergoing elective surgery for benign as well as malign diseases of the pancreatic body and tail. After screening for eligibility and obtaining informed consent, a total of 294 adult patients will be preoperatively randomised in a 1:1 ratio. The primary hypothesis is that MIDP is non-inferior to open distal pancreatectomy in terms of postoperative mortality and morbidity expressed as the Comprehensive Complication Index (CCl) within 3 months after index operation, with a non-inferiority margin of $7.5 \mathrm{CCl}$ points. Secondary endpoints include pancreas-specific complications, oncological safety and patient reported outcomes. Follow-up for each individual patient will be 2 years.

Ethics and dissemination The DISPACT-2 trial has been approved by the Ethics Committee of the medical faculty of Heidelberg University (S-693/2017). Results of the primary endpoint will be available in 2024 and will be published at national and international meetings. Full results will be made available in an open access, peer-reviewed journal. The website www.dispact.de contains up-to-date information regarding the trial.

Trial registration number DRKS00014011

\section{INTRODUCTION}

Resections of the pancreas on the left of the superior mesenteric vein are defined as distal pancreatectomies (DPs). Most resections are performed electively, for causes ranging

\section{Strengths and limitations of this study}

- For the first time, morbidity and mortality will be evaluated in a confirmatory setting for minimally invasive distal pancreatectomy.

- The trial also evaluates primary endpoints from other complete or currently recruiting randomisedcontrolled trials on minimally invasive vs open distal pancreatectomy.

- If minimally invasive distal pancreatectomy proves to be non-inferior to open distal pancreatectomy, the minimally invasive approach may become the recommendation due to its advantages regarding enhanced patient recovery.

- One limitation is that patients with locally advanced disease must be excluded, thus reducing the generalisability of the results. However, most locally advanced cases are treated with open surgery in any case.

from chronic pancreatitis $(24 \%)$ and other benign diseases (22\%) to malignant diseases $(18 \%)$ or neuroendocrine tumours $(14 \%){ }^{1}$ The postoperative mortality rate is below $5 \%$ in high-volume centres, although morbidity has remained largely unchanged for years, and ranges from $30 \%$ to $40 \% .^{1}$ Open DP (ODP) is the gold standard for the surgical treatment of diseases of the pancreatic body and tail. One of the most serious complications following DP is postoperative pancreatic fistula (POPF), where digestive enzymes run into the abdominal cavity from the resected surface, leading to pain, infection and postpancreatectomy haemorrhage. ${ }^{2}{ }^{3}$ While different resection and closure techniques for DP exist, POPF can occur irrespective of which technique is used. ${ }^{4}$ To achieve 
transparency and comparability among different studies, consensus definitions for these complications have been introduced and classifications of surgical complications as well as the Comprehensive Complication Index (CCI) established. $^{23}{ }^{5-9}$ Patient-related outcomes are not only affected by underlying diseases, comorbidities, type of surgical resection, concomitant treatment and several other factors, but also by which approach to the abdominal cavity is taken.

The first DISPACT trial, published in 2011, compared the outcome of stapled DP to that of a conventional scalpel resection with a suture closure of the pancreatic remnant. No significant difference in pancreatic fistula rate was found. ${ }^{10}$ All patients had been operated on by the open abdominal approach.

Minimally invasive surgery has become a standard technique for several abdominal surgical procedures. ${ }^{11-14}$ There is an increasing tendency to use minimally invasive DP (MIDP) in the treatment of pancreatic lesions located in the body and tail. ${ }^{15-17}$ Nevertheless, a minimally invasive resection of pancreatic lesions is still considered controversial, due to some challenges and limitations it poses, including major vessel proximity and pancreas's retroperitoneal location. ${ }^{11}{ }^{18-20}$ Several systematic reviews (SRs) have compared MIDP and ODP in recent years. These SRs underlined the need for well-designed randomised controlled trials (RCTs). ${ }^{21-25}$ To date, two of these RCTs ${ }^{26}{ }^{27}$ have been published and three are actively recruiting. The characteristics and outcomes of these trials are summarised in table 1 . Despite the presence of soft endpoints like length of hospital stay and time to functional recovery, as well as ongoing trials on oncological safety, the missing link here is the proof of surgical safety.

\section{Aim of this study}

The primary hypothesis is that MIDP is non-inferior to ODP in terms of postoperative mortality and morbidity expressed as the CCI within 3 months after index operation with a non-inferiority margin of 7.5 CCI points.

\section{METHODS AND ANALYSIS}

\section{Trial design}

DISPACT-2 trial is an RCT, non-inferiority, multicentre surgical trial with two parallel study groups. Patients planned for an elective DP will be consecutively assessed for eligibility and will be informed about the trial in detail. After providing written informed consent, 294 patients will be preoperatively allocated in a 1:1 ratio to either the minimally invasive or the open arm. The primary outcome parameter will be CCI within 3 months after index operation. Figure 1 shows the patient flow.

\section{Population and eligibility criteria}

To accurately represent DP patients as a whole, and to attain high external validity, broad inclusion criteria that incorporate all diseases of the pancreatic body and tail were chosen. Patients with a high suspicion of irresectability will be excluded. Thus, patients with CA 19-9 levels higher than $1000 \mathrm{UI} / \mathrm{ml}$ and those with preoperative suspicion for distant organ metastases as well as tumour infiltration of the superior mesenteric artery, superior mesenteric vein or hepatic artery will be excluded. Prior major open upper abdominal surgery is confounding, due to the possibility that adhesions in the abdominal cavity requiring adhesiolysis or further intraoperative intervention can in turn cause additional complications that could distort the results. Therefore, these patients will also be excluded. All inclusion and exclusion criteria are displayed in box 1. Patients are free to leave the trial at any time and need not provide a reason for their decision.

\section{Sample size}

Sample size was calculated according to a non-inferiority design, with CCI as the primary endpoint. Assumptions were made based on the results of in-house calculations of CCI from the data of the original DISPACT trial with a mean CCI of 30 and a standard deviation (SD) of 20 points. ${ }^{10} \mathrm{~A}$ decrease of CCI by 10 points or more is considered clinically relevant by patients as well as physicians. ${ }^{9}{ }^{28}$ A margin of 7.5 CCI points will be tolerated because it corresponds to the occurrence of less than one major

Table 1 RCTs investigating MIDP versus ODP

\begin{tabular}{|c|c|c|c|c|c|}
\hline Terminated trials & Year & $\operatorname{MIDP}(n)$ & $\begin{array}{l}\text { Robotic } \\
\text { assisted }\end{array}$ & ODP (n) & Primary endpoint \\
\hline LEOPARD (REF) & 2018 & 51 & yes & 57 & $\begin{array}{l}\text { Time to functional recovery: MIDP } 4 \text { days (IQR } 3-6 \text { ) } \\
\text { vs ODP } 6 \text { days (IQR } 5-8) ; p<0.001\end{array}$ \\
\hline LAPOP (REF) & 2020 & 29 & no & 29 & $\begin{array}{l}\text { Length of postoperative hospital stay: MIDP } 5 \text { days } \\
\text { (IQR 4-5) vs ODP } 6 \text { days (IQR 5-7); } p=0.002\end{array}$ \\
\hline Ongoing trials & $\begin{array}{l}\text { Termination } \\
\text { expected }\end{array}$ & $\begin{array}{l}\text { Sample } \\
\text { size }\end{array}$ & $\begin{array}{l}\text { Robotic } \\
\text { assisted }\end{array}$ & Hypothesis & Primary endpoint \\
\hline NCT04483726 & 2022 & 258 & yes & Non-inferiority & Microscopically radical resection rate \\
\hline NCT03792932 & 2022 & 306 & no & Superiority & 2-year recurrence-free survival \\
\hline NCT03957135 & 2025 & 244 & no & Non-inferiority & 2-year overall survival \\
\hline
\end{tabular}

MIDP, minimally invasive distal pancreatectomy; ODP, open distal pancreatectomy; RCTs, randomised controlled trials. 


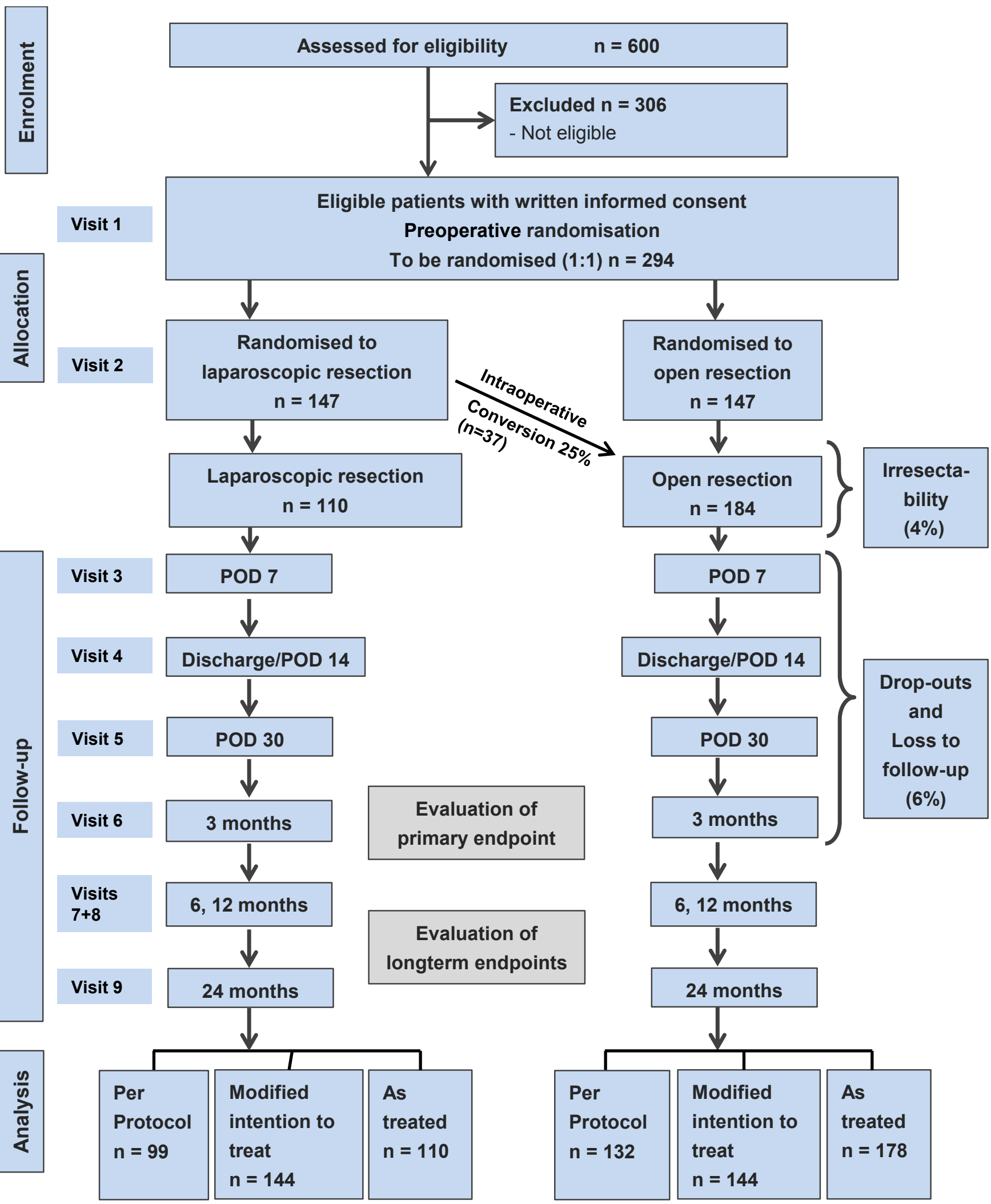

Figure 1 CONSORT flow chart. CONSORT, Consolidated Standards of Reporting Trials; POD, postoperative day.

complication, that is, a difference that is not clinically relevant. In sum, a mean CCI of 30 points in both the intervention and control groups, with an SD of 20 points and a non-inferiority margin of 7.5 points, are assumed.
The primary analysis will be based on three different analysis sets: the modified intention to treat (mITT) set, the per protocol (PP) set and the as treated (AT) set. $^{29}$ The sample size is calculated to achieve 


\section{Box 1 Inclusion and exclusion criteria}

- Planned distal pancreatectomy with or without splenectomy for any indication.

- Patient suitable for both surgical techniques.

Age $\geq 18$ years.

- Ability of subject to understand character and individual consequences of the clinical trial.

- Written informed consent.

- Patients scheduled for a pancreatic resection other than distal pancreatectomy.

- Distant organ metastases.

- Tumour infiltration of the superior mesenteric vein or superior mesenteric artery or hepatic artery or infiltration of adjacent organs.

- CA 19-9>1000 IU $/ \mathrm{mL}$.

- $\mathrm{ASA}>3$.

- Prior major open upper abdominal surgery.

Left-sided portal hypertension.

- Participation in another intervention-trial with interference of intervention and/or outcome of this study.

Expected lack of compliance

$80 \%$ power when analysing the smallest population (PP set).

Patients converted from minimally-invasive to open surgery will not be included in the PP set. As the randomisation will be carried out with a 1:1 ratio and a maximum conversion rate from MIDP to ODP of $25 \%$ is assumed, this results in a ratio of up to 3:4 in the PP set. Given these assumptions, applying a one-sided t-test at a onesided significance level of $0.025,99$ patients need to be analysed in the MIDP group and 132 patients in the ODP group (calculations were made using Nquery V.7.0.). ${ }^{30}$ With a lower conversion rate than the assumed $25 \%$, the MIDP group in the PP set will be larger, leading to a slight increase in power of the t-test. In addition, performing the primary analysis with a linear mixed model, including centre as random intercept leads to an equal or even increased power compared with using a one-sided t-test. To compensate for non-resectable patients not included in the primary analysis and drop-outs/lost to-follow-ups, a further $10 \%$ of patients will be randomised, resulting in 110 patients in the MIDP arm and 147 patients in the ODP arm. Given the planned randomisation ratio of 1:1, 147 patients must be included into each arm, resulting in a total of 294 patients to be randomised.

\section{Trial-specific procedures}

The DISPACT-2 trial compares MIDP as an experimental procedure to ODP as a control procedure. MIDP may be performed via a conventional laparoscopic or roboticassisted approach.

\section{Experimental intervention-MIDP}

In some DISPACT-2 trial centres, patients are offered both robotic-assisted surgery and the conventional laparoscopic approach. Other centres only offer conventional laparoscopy. None of the centres offer exclusively roboticassisted surgery. After randomisation to the minimally invasive treatment group, the use of conventional laparoscopy or robotic-assisted surgery is up to the participating surgeon. According to the surgeon's preference, 3-4 abdominal trocars will be placed. One $12 \mathrm{~mm}$ trocar will be placed for the camera beneath the umbilicus and a pneumoperitoneum is created. Then, the lesser sac is entered and a lateral to medial dissection is performed below the inferior border of the pancreatic tail. The splenic flexure is mobilised to expose the inferior edge of the pancreatic tail. By incising the peritoneum from the inferior edge of the pancreas to the inferior pole of the spleen, the pancreas is then mobilised from the retroperitoneum. In the case of splenic preservation, the splenic vessels need to be spared. The pancreas is divided using a stapler. If transection with a stapler is impossible, the surgeon is free to convert to open surgery or to perform any other transection technique. The specific techniques used will be recorded. In case of splenectomy, the organ is dissected, placed in an extraction bag, and removed through an extension of one of the $12 \mathrm{~mm}$ trocar sites or a Pfannenstiel incision.

Whenever conversion to an open procedure is necessary, this leads to a crossover of patients from MIDP to ODP, so events resulting in conversion (eg, significant bleeding) will be recorded in detail.

\section{Control intervention-ODP}

A midline or transverse incision will be performed according to surgeon's preference. The lesser sac is entered and a lateral to medial dissection below the inferior border of the pancreatic tail is performed. The splenic flexure is mobilised to expose the inferior edge of the tail of the pancreas, which is then mobilised by incising the peritoneum from the inferior pancreatic edge to the inferior pole of the spleen. In the case of splenic preservation, the splenic vessels need to be spared. Pancreatic transection should be performed either by stapler or by scalpel and followed by a suture closure of the pancreatic remnant. The surgeon should perform the transection technique with which he or she is most experienced.

\section{Concomitant treatment}

Due to the pragmatic nature of the DISPACT-2 trial, only minimal effort will be made to standardise concomitant treatments. Centres will use whatever materials and medical devices are most used in their daily practice. However, all surgical techniques, materials, and medical devices used will be reported in detail to detect any differences among centres, as well as to identify potential confounders and to register any imbalance among the treatment groups.

Any additional treatment of the pancreatic remnant, as well as details of the resection procedure, including performance of spleen preservation and, especially, drain management, will be reported in detail. If a drain is placed, early removal (on postoperative day 1-3) is encouraged. Perioperative use of somatostatin or its analogues will also be recorded. 


\section{Data capture and trial endpoints}

For each included patient the following baseline parameters will be assessed during the first trial visit, after a patient has given written informed consent: age (years), gender (male/female/diverse), height $(\mathrm{cm})$, weight $(\mathrm{kg})$, current employment status (employed, housewife/househusband, unemployed, retired), smoker (yes/no), underlying disease (malignant/chronic pancreatitis/other), neoadjuvant therapy within the last 6 months (radiotherapy/chemotherapy), current medication within 2 weeks before surgery, prior imaging studies, American Society of Anesthesiologists (ASA) classification (I-IV), updated Charlson Comorbidity Index (all items) and Quality of life questionnaires of the European Organisation for Research and Treatment of Cancer (EORTC QLQ-C30, PAN28).

Intraoperatively, data on operative time (minutes), surgeon experience (1-20/21-50/51-100/>100 MIDP resections; $1-20 / 21-50 / 51-100 />100$ open distal pancreatic resections), blood-loss (mL), drain procedures (yes/ no), concomitant treatments, type of surgical intervention (robotic assisted, laparoscopic, open), need for conversion (yes/no), type of extraction incision for MIDP, mode of access for ODP (midline, transverse incision), tumour localisation, vascular involvement, extent of resection (and a detailed report of the resection process), pancreas texture, diameter of the pancreatic duct, and any histopathological findings will be gathered.

\section{Primary endpoint}

Overall postoperative morbidity within 3 months after index operation assessed as CCI was chosen as the primary endpoint since this parameter considers the patient's perspective as well as parameters of surgical effectiveness. ${ }^{9}$

\section{Determination of primary endpoint}

The CCI has values ranging from 0 to 100 . An index of 0 indicates no complications at all whereas 100 means the patient has died. Between 0 and 100, the index expresses the patient's cumulative morbidity. The CCI is based on the established Clavien-Dindo classification, ${ }^{7}$ which divides complications into five grades from I to $\mathrm{V}$ and includes four subclasses (IIIa/ IIIb and IVa/ IVb). A complication is defined as any deviation from the normal postoperative course. Examples of complications in different classes are superficial surgical site infection with bedside treatment (class I), pneumonia in need of antibiotic treatment (class II), POPF in need of drainage guided by computed tomography (CT) (class IIIa) or reoperation (class IIIb), single (class IVa) or multiple organ failure (class IVb) and death (class V). Because all complications are summed in the CCI, complications of a more severe class result in a higher CCI. The index is constructed to be approximately normally distributed. ${ }^{9}$ The score is validated for the pancreatic surgical population and a difference of 10 is regarded as a clinically relevant difference. ${ }^{28}$ An endpoint like the CCI, which reflects the entire spectrum of possible complications, is highly appropriate for comparing minimally invasive and open surgical approaches to the abdominal cavity. For the present study, CCI will be calculated for all complications within 3 months after index operation.

\section{Secondary endpoints}

Secondary endpoints will include perioperative, postoperative and long-term endpoints (table 2). If available, validated definitions e.g. by the International Study Group of Pancreatic Surgery (ISGPS) or the Center for Disease Control and Prevention (CDC) will be used.

\section{Patient timeline and description of trial visits}

All patients scheduled for elective DP will be screened consecutively and subjected to a screening list. Reasons for non-enrolment must be stated. Patients will be enrolled given their ability to understand the extent and nature of the trial, to provide written informed consent after receiving detailed information, and by fulfilling all inclusion criteria. Baseline data will be collected during the screening/baseline visit (visit 1 ). The above-mentioned surgical data will be collected during visit 2. Primary and secondary outcome parameters will be collected during visits 3-9, whereby visits $5-9$ will be performed via telephone. Furthermore, diagnostic and any ensuing therapeutic procedures caused by postoperative complications will be collected and reported. Table 3 summarises the visits.

\section{Randomisation and allocation}

In order to obtain equally sized intervention groups, patients will be allocated after the screening visit by using a concealed centralised web-based tool (randomizer. at), in a 1:1 ratio. Randomising patients before the day of surgery is justifiable for practical reasons, such as the need to prepare a specific operating theatre or different devices for a minimally invasive versus open approach. Block randomisation of variable sizes will be performed. Centres will be stratified according to enrolment (all centres that reported to recruit fewer than 25 patients will be grouped together and each of the seven larger centres will constitute a stratum). Small centres will be grouped together because the total number of centres is relatively large in relation to the number of patients.

\section{Blinding}

Blinding will be reported according to standards of surgical trial methodology. ${ }^{31}$ Blinding of the operating surgeon is not possible. Patients are blinded to the intervention for as long as possible. Postoperative blinding of patients, for example, by large patches, is not feasible, since unblinding during change of wound dressings would take place prior to the assessment of the primary endpoint ( 3 months postoperatively). Similarly, blinded outcome collection of postoperative complications is not feasible. However, assessment of CCI will be conducted blinded, that is, the responsible investigator will receive documents masking the intended or actual allocation when forming conclusions about the presence or severity 
Table 2 Secondary endpoints

\begin{tabular}{|c|c|c|}
\hline Endpoint & Definition & Time point \\
\hline \multicolumn{3}{|l|}{ Perioperative endpoints } \\
\hline Operative time & Minutes & Day of operation \\
\hline Intraoperative blood loss & Millilitres & Day of operation \\
\hline Conversion rate & $\begin{array}{l}\text { Conversion to open surgery after starting } \\
\text { minimally-invasive surgery }\end{array}$ & Day of operation \\
\hline \multicolumn{3}{|l|}{ For oncological patients } \\
\hline Resection rate & R0/R1 resection ${ }^{38}$ & Day of operation \\
\hline Resected lymph nodes & According to pathologist & Day of operation \\
\hline Positive lymph nodes & According to pathologist & Day of operation \\
\hline
\end{tabular}

Postoperative endpoints

Pancreas-associated morbidity within 3 months

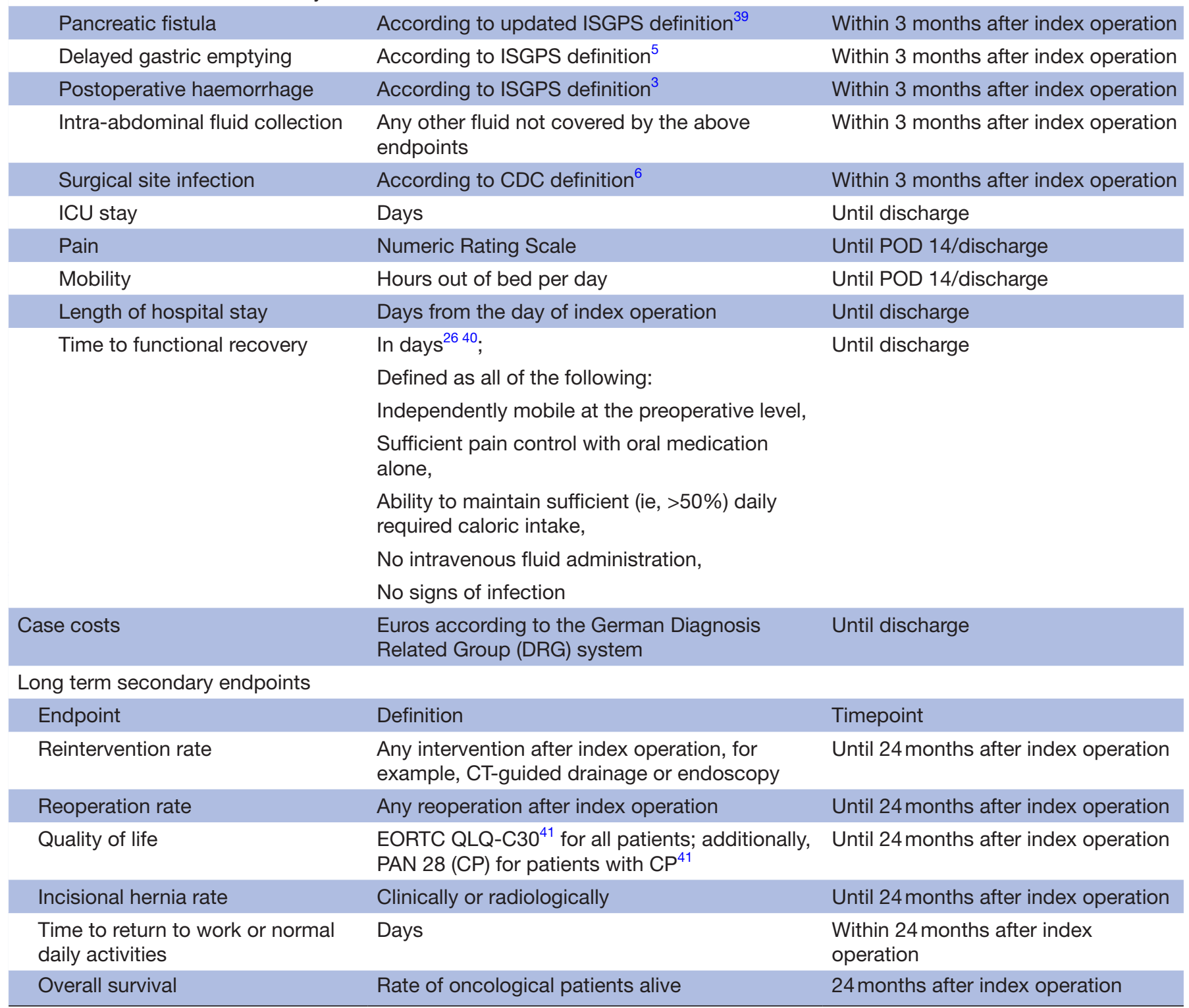

$\mathrm{CP}$, chronic pancreatitis.

of complications, for example, of a pancreatic fistula. Therefore, outcome assessment will be as free of detection bias as possible. No attempts will be made to blind the trial statisticians; however, they will not have access to unblinded data during the study and will perform 
Table 3 Trial visits and documented parameters

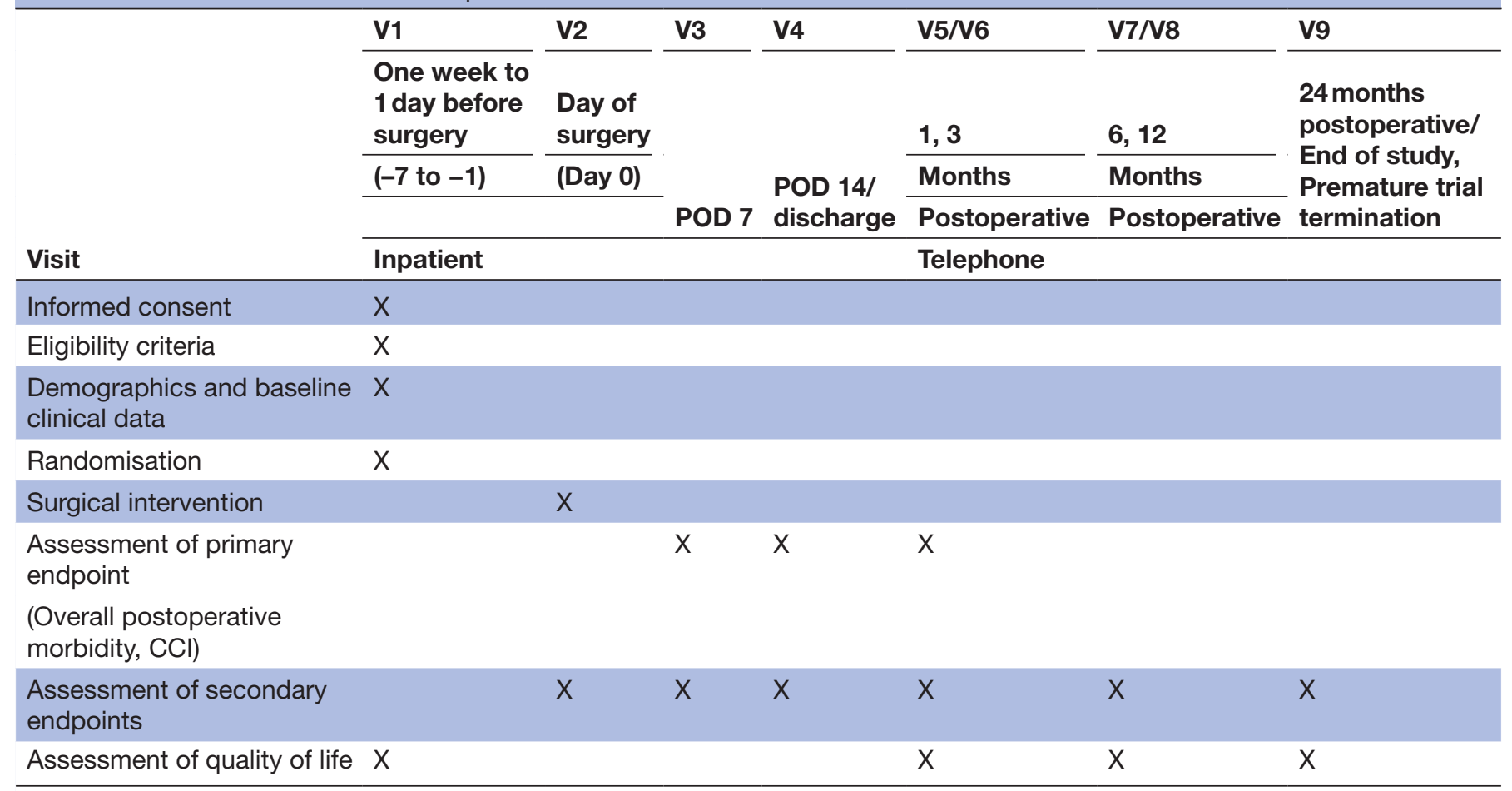

$\mathrm{CCl}$, Comprehensive Complication Index; POD, postoperative day.

analyses according to a predefined statistical analysis plan, which will be finalised prior to database closure at latest.

\section{Other methods for minimising bias}

Minimising attrition bias

Statistical methods such as multiple imputation will be used to minimise risk of bias due to incomplete outcome data as outlined below. Structured monitoring as outlined below will further minimise attrition bias.

\section{Minimising reporting bias}

The results will be reported according to the Consolidated Standards of Reporting Trials statement. ${ }^{32}$ In order to assure full transparency throughout this trial and its subsequent reporting, the trial protocol is, according to the Standard Protocol Items: Recommendations for Interventional Trials statement, ${ }^{33}$ hereby published.

\section{Minimising other forms of bias}

This is an investigator-initiated trial funded by the Deutsche Forschungsgemeinschaft / German Research Foundation (DFG) - PR 1651/1-1. Any financial relationship with medical device providers or conflicts of interest that could inappropriately influence work within this project will be stated explicitly.

\section{Assessment of cross-over patients (conversions from MIDP to} ODP)

The definite rate of conversion from MIDP to ODP cannot be foreseen, nor can conversion be completely avoided by modifying inclusion and exclusion criteria, since reasons for conversion arise intraoperatively, for example, for technical infeasibility, significant bleeding. Occasional conversion from MIDP to ODP reflects clinical reality and will not vanish even after completion of learning curves. With respect to conversion rates during MIDP, the current available evidence is insufficient. Therefore, the rate of patients who will require conversion from MIDP to ODP within this trial is estimated cautiously at up to $25 \%$. Reasons for conversion will be captured for further evaluation of this specific subgroup, thus allowing potential differences among the outcomes of minimally invasive, open and converted surgery to be detected and described.

\section{Data management, statistics and quality assurance} Data management

All protocol-required information will be entered by the investigator or designated representative in the electronic case report form (eCRF) as soon as possible after it has been collected. Any outstanding entries will be completed immediately after the final visit. An explanation should be given for all missing data. Any entry and correction in the remote data entry system will be protocolled automatically in an audit file.

The completed eCRF must be reviewed and signed by the investigator. Validating programmes will examining the completeness, validity and plausibility of data by generating queries that will then be clarified by the investigator or designated representatives.

\section{Statistical methods}

Non-inferiority of MIDP versus ODP will be assessed using a linear mixed model including centre as random 
intercept and group as fixed effect. The grouping of centres used in the randomisation process was based primarily on practical, rather than clinical, reasons (the relatively large number of centres in relation to number of patients). While differences between centres cannot be ruled out, they are of no special interest for the primary analysis, so inclusion of centre as a random effect is recommended. ${ }^{34}$ The one-sided significance level is set to $2.5 \%$. The hypotheses to be assessed in the primary efficacy analysis are as follows: H0: $\mu \mathrm{MIDP}-\mu \mathrm{ODP} \geq \delta$ and $\mathrm{H} 1: \mu \mathrm{MIDP}-\mu \mathrm{ODP}<\delta$, where $\delta=7.5$ represents the chosen non-inferiority margin. $\mu \mathrm{MIDP}$ and $\mu \mathrm{ODP}$ denote the mean CCI in the MIDP and ODP groups, respectively. The primary efficacy analysis will be based on three analysis sets. Patients deemed unresectable during surgery will not be considered in any of the analysis sets, as the primary aim is to estimate the intervention effect after at least a partial resection of the pancreas. It can be assumed that the proportion of non-resectable patients is comparable in both groups (as randomised). In the MIDP group, patients are very likely to be switched to open surgery before non-resectability is decided on. The PP set consists of all patients treated PP. Patients with major protocol violations and converted patients will be excluded and no missing data will be imputed (non-resectable patients not considered). The mITT set comprises all patients in the group to which they were randomised (converted patients remain in the MIDP group; non-resectable patients not considered). In addition, the AT set will be analysed separately, considering patients according to their final, rather than randomised, treatment (converted patients in the ODP group; non-resectable patients not considered). There is evidence that converted patients will have a higher postoperative CCI than those patients randomised to the ODP group. ${ }^{35}$ However, this evidence results from retrospective data analysis, leaving room for bias and deviating observations in our prospective randomised study. In any case, the AT set is an important complement to the two common (and recommended) analysis sets here. For robust interpretation, the results of the three primary analysis sets should lead to similar conclusions ${ }^{29}$; otherwise, possible reasons must be discussed. Analysing the mITT set can be interpreted as a treatment policy approach, according to the estimands framework. ${ }^{33}$ Nonresectability is handled by excluding those patients from the analysis as they are not part of the targeted population. Other post-randomisation events, like resection of adjacent organs, will be ignored.

In the mITT and AT sets, missing data for the primary outcome variable will be imputed. It can be assumed that information regarding CCI will be complete by the time of discharge. Missing information after discharge will be replaced by the mean value in the respective time window (eg, between visits 4 and 5) of the corresponding treatment group. To assess the robustness of the results, bestcase and worst-case scenarios will be evaluated.

A focus will be given to the description and comparison of patient characteristics and outcomes of the converted patients in comparison to the patients treated in the MIDP group as randomised and the patients randomised to ODP.

In general, all baseline values and secondary outcomes will be evaluated descriptively, with descriptive $p$ values reported along with 95\% CIs for the corresponding effects. Secondary endpoints will be also evaluated descriptively, using regression models including group as fixed effect and centre as random intercept as specified for the primary endpoint. Time-to-event endpoints will be evaluated by methods of survival analysis including Cox proportional hazards models for oncological patients. Confounding factors should be equally distributed by randomisation; however, known factors influencing postoperative complications will be recorded and compared among the groups. These confounding factors include baseline patient characteristics, pancreatic texture and duct size, closure technique of the pancreatic remnant and splenic preservation. In further exploratory analyses, any association between the above-mentioned factors and the primary and selected secondary outcomes will be assessed. In addition, subgroup analyses (eg, oncological vs non-oncological patients, laparoscopic vs roboticassisted surgery in the MIDP group, or patients who switched from MIDP to ODP) will be carried out. Serious adverse events and adverse events are collected as minor and major complications throughout the study and will be tabulated as rates with $95 \%$ CIs. Statistical methods will be used to assess the quality of data and the homogeneity of the intervention groups.

Graphical methods (eg, boxplots, Kaplan-Meier plots) will be used to visualise findings. All analyses will be done using SAS V.9.4 or higher.

\section{Quality assurance}

Independent monitors of the Study Center of the German Society of Surgery (SDGC) will perform risk-based clinical monitoring according to its standard operating procedures. All investigators will grant monitors access to trialspecific patient data and agree to being visited before, during and after completion of the study to ensure that the study is conducted, recorded and reported on according to the study protocol, Good Clicical Practice (GCP) requirements, and all applicable laws and regulations (eg, data protection). The monitoring strategy will consist of a combination of centralised and on-site monitoring. On-site monitoring will focus on patient informed consent and safety, inclusion and exclusion criteria, surgical procedures, randomisation, and correct recording and documentation of primary and secondary endpoints by source data verification.

\section{Duration and schedule}

The duration of the trial for each patient is 24 months. The overall trial is expected to take 5 years to complete, including study preparation and analysis. The first patient was recruited in August 2020, at the University of 
Heidelberg. While study completion is expected by late 2025 , the actual overall duration or recruitment time may differ.

\section{Ethics}

Both surgical procedures in this proposed trial represent current clinical practice. Only patients equally suited for both surgical techniques will be eligible for this trial. During minimally invasive surgery, a conversion to open distal pancreatic resection may be indicated due to intraoperative problems such as technical problems or bleeding. This procedure represents current clinical practice and patients will be informed preoperatively about this possibility. To summarise, no increased medical risks for the participating patients are expected.

All patients will be informed both orally and in writing by a trained and authorised investigator about the aims of the study, the possible risks, the procedures and possible hazards to which he/she will be exposed, and the mechanism of treatment allocation. It will be emphasised that participation is voluntary and that the patient can withdraw from the trial whenever he/she wants to, for any reason. This will not prejudice the patient's subsequent care. All patients will be given ample time to decide whether or whether not they wish to participate in the trial.

This study will be conducted in accordance with the Declaration of Helsinki, ${ }^{36}$ the international principles of GCP (ICH-GCP E6 guidelines), where appropriate for a non-pharmacological trial, the Medical Association's professional code (Berufsordnung der Bundesärztekammer) $\$ 15$ (non-AMG/non-MPG trials) and all other applicable laws, for example, data protection law and the European General Data Protection Regulation. The protocol was reviewed and approved by the Ethics Committee of the medical faculty at the University of Heidelberg (S-693/2017) and serves as the primary ethics committee for this trial. In all participating centres, the local ethics committee must provide additional consent.

\section{Patient involvement}

The Department of General, Visceral and Transplantation Surgery at the University of Heidelberg performed Germany's first priority setting partnership for pancreatic cancer. This project involved all relevant stakeholders (including patients, their families and their caregivers) in identifying the most pressing unanswered research questions in pancreatic cancer. Among the top three questions, finding the best treatment ranked first and treating surgical complications ranked third. ${ }^{37}$ Moreover, patients were involved in developing the CCI, the primary endpoint of this trial. Furthermore, this trial also investigates patient-reported outcomes (QoL).

Finally, a representative of pancreatectomy patients (Arbeitskreis der Pankreatektomierten e.V.) will be a member of the data safety and monitoring board. Therefore, this study continuously takes the patient's perspective into account, and across a variety of platforms.

\section{Dissemination}

The study website (www.dispact.de) contains all up-todate information regarding the trial. Final trial results will be published in a peer-reviewed journal. Furthermore, results will be presented at appropriate national and international conferences. After publication, the anonymised data set will be made available, on request, for further research by non-participating parties. Relevant information about the trial and its results will be disseminated to patient groups (eg, the German Arbeitskreis der Pankreatektomierten e.V.) and on social media.

\section{Author affiliations}

${ }^{1}$ Department of General, Visceral and Transplantation Surgery, University of Heidelberg, Heidelberg, Germany

${ }^{2}$ The Study Center of the German Society of Surgery (SDGC), University of Heidelberg, Heidelberg, Germany

${ }^{3}$ Institute of Medical Biometry and Informatics, University of Heidelberg, Heidelberg, Germany

Acknowledgements This paper was edited for language by Elizabeth Corrao-Billeter.

Contributors PP, MKD, BPM-S, AM, MWB, CD-H and FS developed the trial concept and wrote the protocol and the manuscript of the protocol publication. IR, FN, HT, AS, TB and CK helped to develop the trial concept and revised the manuscript critically for important intellectual content. All authors approved the final version for publication agreed to be accountable for all aspects of the work and ensure that any questions related to the accuracy or integrity of any part of the work will be appropriately investigated and resolved.

Funding This is an investigator-initiated trial funded by the Deutsche Forschungsgemeinschaft/German research Foundation (DFG)-PR 1651/1-1.

Disclaimer The funding source of the trial had no role in trial design and will not have a role in data collection, analysis, or interpretation of the results, or in the writing of the final report.

Competing interests FN reports receiving travel support for conference participation as well as equipment provided for laparoscopic surgery courses by KARL STORZ, Johnson \& Johnson, Intuitive Surgical, Cambridge Medical Robotics and Medtronic. None of the other authors has a secondary interest according to the ICMJE guidelines that could inappropriately influence their contributions to this work.

Patient and public involvement Patients and/or the public were involved in the design, or conduct, or reporting, or dissemination plans of this research. Refer to the Methods section for further details.

Patient consent for publication Not required.

Provenance and peer review Not commissioned; peer reviewed for ethical and funding approval prior to submission.

Open access This is an open access article distributed in accordance with the Creative Commons Attribution Non Commercial (CC BY-NC 4.0) license, which permits others to distribute, remix, adapt, build upon this work non-commercially, and license their derivative works on different terms, provided the original work is properly cited, appropriate credit is given, any changes made indicated, and the use is non-commercial. See: http://creativecommons.org/licenses/by-nc/4.0/.

\section{ORCID iDs}

Pascal Probst http://orcid.org/0000-0002-0895-4015

Arianeb Mehrabi http://orcid.org/0000-0001-6163-1525

\section{REFERENCES}

1 Lillemoe KD, Kaushal S, Cameron JL, et al. Distal pancreatectomy: indications and outcomes in 235 patients. Ann Surg 1999;229:693-8.

2 Bassi C, Dervenis C, Butturini G, et al. International Study Group on pancreatic fistula definition: postoperative pancreatic fistula: an international Study Group (ISGPF) definition. Surgery 2005;138:8-13. 
3 Wente MN, Veit JA, Bassi C, et al. Postpancreatectomy hemorrhage (PPH): an international Study group of pancreatic surgery (ISGPS) definition. Surgery 2007;142:20-5.

4 Probst $\mathrm{P}$, Hüttner FJ, Klaiber U, et al. Stapler versus scalpel resection followed by hand-sewn closure of the pancreatic remnant for distal pancreatectomy. Cochrane Database Syst Rev 2015;11:CD008688.

5 Wente MN, Bassi C, Dervenis C, et al. Delayed gastric emptying (DGE) after pancreatic surgery: a suggested definition by the International Study group of pancreatic surgery (ISGPS). Surgery 2007;142:761-8.

6 Horan TC, Gaynes RP, Martone WJ, et al. Cdc definitions of nosocomial surgical site infections, 1992: a modification of CDC definitions of surgical wound infections. Infect Control Hosp Epidemiol 1992;13:606-8.

7 Dindo D, Demartines N, Clavien P-A. Classification of surgical complications: a new proposal with evaluation in a cohort of 6336 patients and results of a survey. Ann Surg 2004;240:205-13.

8 Koch M, Garden OJ, Padbury R, et al. Bile leakage after hepatobiliary and pancreatic surgery: a definition and grading of severity by the International Study group of liver surgery. Surgery 2011;149:680-8.

9 Slankamenac K, Graf R, Barkun J, et al. The comprehensive complication index: a novel continuous scale to measure surgical morbidity. Ann Surg 2013;258:1-7.

10 Diener MK, Seiler CM, Rossion I, et al. Efficacy of stapler versus hand-sewn closure after distal pancreatectomy (DISPACT): a randomised, controlled multicentre trial. Lancet 2011;377:1514-22.

11 Teh SH, Tseng D, Sheppard BC. Laparoscopic and open distal pancreatic resection for benign pancreatic disease. J Gastrointest Surg 2007;11:1120-5.

12 Ammori BJ, El-Dhuwaib Y, Ballester P, et al. Laparoscopic distal pancreatectomy for neuroendocrine tumors of the pancreas. Hepatogastroenterology 2005;Mar-Apr;52:620-4.

13 Sain AH. Laparoscopic cholecystectomy is the current "gold standard" for the treatment of gallstone disease. Ann Surg 1996;224:689-90.

14 Smith CD, Weber CJ, Amerson JR. Laparoscopic adrenalectomy: new gold standard. World J Surg 1999;23:389-96.

15 Goh BKP, Tan YM, Chung YF. Critical appraisal of 232 consecutive distal Pancreatectomies with emphasis on risk factors, outcome and management of the postoperative pancreatic fistula. Arch Surg 2008;143:956-65.

16 Luo Y, Liu R, Hu M-G, et al. Laparoscopic surgery for pancreatic insulinomas: a single-institution experience of 29 cases. $J$ Gastrointest Surg 2009;13:945-50.

17 Vezakis A, Davides D, Larvin M, et al. Laparoscopic surgery combined with preservation of the spleen for distal pancreatic tumors. Surg Endosc 1999;13:26-9.

18 Andrén-Sandberg Åke, Wagner M, Tihanyi T, et al. Technical aspects of left-sided pancreatic resection for cancer. Dig Surg 1999;16:305-12.

19 Addeo P, Giulianotti PC. Update on laparoscopic pancreatectomy in 2010. Minerva Chir 2010;65:655-66.

20 Merchant NB, Parikh AA, Kooby DA. Should all distal pancreatectomies be performed laparoscopically? Adv Surg 2009;43:283-300.

21 Jusoh AC, Ammori BJ. Laparoscopic versus open distal pancreatectomy: a systematic review of comparative studies. Surg Endosc 2012;26:904-13.
22 Sui C-J, Li B, Yang J-M, et al. Laparoscopic versus open distal pancreatectomy: a meta-analysis. Asian J Surg 2012;35:1-8.

23 Venkat R, Edil BH, Schulick RD, et al. Laparoscopic distal pancreatectomy is associated with significantly less overall morbidity compared to the open technique. Ann Surg 2012;255:1048-59.

24 Pericleous S, Middleton N, McKay SC, et al. Systematic review and meta-analysis of case-matched studies comparing open and laparoscopic distal pancreatectomy. Pancreas 2012;41:993-1000.

25 Jin T, Altaf K, Xiong JJ, et al. A systematic review and meta-analysis of studies comparing laparoscopic and open distal pancreatectomy. HPB 2012:14:711-24.

26 de Rooij T, van Hilst J, van Santvoort H. Dutch pancreatic cancer Group. minimally invasive versus open distal pancreatectomy (leopard): a multicenter patient-blinded randomized controlled trial. Ann Surg 2019;269:2-9.

27 Björnsson B, Larsson AL, Hjalmarsson C, et al. Comparison of the duration of hospital stay after laparoscopic or open distal pancreatectomy: randomized controlled trial. Br J Surg 2020;120.

28 Slankamenac Ket al. The comprehensive complication index: a novel and more sensitive endpoint for assessing outcome and reducing sample size in randomized controlled trials. Ann Surg 2014;260:757-62.

29 EMEA. CPMP/EWP/482/99: points to consider on switching between superiority and non-inferiority. London: EMEA, 2000.

30 nQuery. Sample size and power calculation. "statsols". Cork, Ireland: Statistical Solutions Ltd, 2017.

31 Probst P, Zaschke S, Heger P, et al. Evidence-Based recommendations for blinding in surgical trials. Langenbecks Arch Surg 2019;404:273-84.

32 CONSORT. Welcome to the CONSORT website, 2020. Available: www.consort-statement.org

33 SPIRIT. Guidance for clinical trial protocols, 2020. Available: http:// www.spirit-statement.org/

34 Kahan BC, Harhay MO. Many multicenter trials had few events per center, requiring analysis via random-effects models or GEEs. J Clin Epidemiol 2015;68:1504-11.

35 Stiles ZE, Dickson PV, Deneve JL, et al. The impact of unplanned conversion to an open procedure during minimally invasive pancreatectomy. J Surg Res 2018;227:168-77.

36 World Medical Association. World Medical association Declaration of Helsinki: ethical principles for medical research involving human subjects. JAMA 2013;310:2191-4.

37 Klotz R, Doerr-Harim C, Ahmed A. Priority setting partnership pancreatic cancer. top ten research priorities for pancreatic cancer therapy. Lancet Oncol 2020;21:e295-6.

38 Strobel O, Hank T, Hinz U, et al. Pancreatic cancer surgery: the new R-status counts. Ann Surg 2017;265:565-73.

39 Bassi C, Marchegiani G, Dervenis C, et al. The 2016 update of the International Study Group (ISGPS) definition and grading of postoperative pancreatic fistula: 11 years after. Surgery 2017;161:584-91.

40 de Rooij T, van Hilst J, Vogel JA, et al. Minimally invasive versus open distal pancreatectomy (leopard): study protocol for a randomized controlled trial. Trials 2017;18:166.

41 Quality of Life. Quality of life group website, 2020. Available: https:// qol.eortc.org 